\title{
Internet-based Education System of Mobile Cloud Environment
}

\author{
Junying Liu \\ Training department, Shijiazhuang Posts and Telecommunications Technical \\ College \\ ljytxh@163.com
}

\begin{abstract}
With the development of mobile Internet, Internet education has entered a new stage of development, but the Internet education system for the mobile Internet, the lack of effective distributed computing capabilities, it allows remote education system, low efficiency of the mobile Internet, business and education means poor, for this problem, we established a mobile Internet-based cloud computing distance education system, it is possible for the mobile Internet network architecture, a reasonable allocation of network resources, cloud computing means to improve the efficiency of the network, distance education has laid a good business diversification Web environment.
\end{abstract}

Keywords: Mobile Internet; Internet Education; Cloud computing; network resources;

\section{Introduction}

With the development of wireless communication technology, particularly mobile cellular network deployment upgrade, so that the video, audio, and other forms of data can be efficiently transmitted. Development of mobile Internet, you can make it anytime, anywhere you can access the Internet, thus enriching the mobile Internet can offer services. Education in the mobile Internet era, education also will be changed, in particular the use of distance education information technology, and its ever-changing business type, and its means of education has been improved to become for all people, anytime, anywhere can be carried out ${ }^{[1-3]}$. However, among the mobile Internet, since the information society, provided data, video data and audio data are many educational resources for each topic are vast, how to choose the best resources for learning and how to identify individual learning route is problems encountered in traditional distance education, but also the movement of distance Education in the Internet environment, especially under conditions of the mobile Internet, the ability to calculate the rate of the intelligent terminal and the last radio transmission limitations, so this problem is more prominent.

Open Gardens for the first time put forward the concept of mobile cloud computing and mobile web users is defined as the application mode to obtain the necessary infrastructure, platform, software and other resources or information services. But distance education mobile Internet environment also have to solve this problem advantages, one is remotely educational mobile Internet environment to participate more, it increases the manual evaluation of the opportunity ${ }^{[4-6]}$, which can SELECTION helpful; Also, because cloud computing the development of technology, can solve the mobile Internet environment, the mobile terminal calculates low capacity bottlenecks.

Although in the past the education system have emphasized shared network, but also the so-called shared at a relatively low level. Modern distance education system there are still many shortcomings, mainly the following: teaching resources and methods, although in various forms, but the format is not uniform, cannot be unified and effective coordination and sharing; hardware and operating systems, various isomers systems coexist, poor compatibility, most of the distance education system and teaching resource 
does not support migration of heterogeneous operating systems and hardware platforms, resulting in a lot of duplication of effort; on the implementation, mostly static or dynamic Web pages, does not reflect the service concept. This is a summary of the application from the shortcomings; however, as new technologies - development of network user behavior analysis, which is the use of a wide range of knowledge on the behavior of Internet users access the full range of analysis, mining analysis of its Internet laws. Network's data network is the basis of the analysis of user behavior. Based on traffic data network traffic monitoring devices that can analyze user sessions and user preference analysis. User Session Analysis for analysis and user related session user-level session information including the session-level analysis. According to this analysis means users can filter out the majority of educational resources are more affected by distance education users welcome.

From the mobile Internet and cloud computing features, the cloud can be used to improve the performance of the mobile terminal as an application-mode mobile Internet environment. The mobile terminal is limited by the size and portability requirements, the processing power, storage space $\mathrm{W}$ and enhance battery life will encounter technical bottlenecks, and cloud computing technology can help break the limit mobile terminal. Users will process and store data after migrating to the cloud, you can weaken the mobile terminal performance requirements. In this mode, the mobile terminal is mainly responsible for interworking with the user, and displays the results from the business cloud on the screen, and complex calculations done by the cloud, so the mobile terminal does not need computing power can have a strong response users of the service request, and provide satisfactory service ${ }^{[7-8]}$.

Although the calculation is based on mobile Internet cloud, hereinafter referred to as mobile cloud computing service model has been proposed based on optimization model and user experience for individual users and business users are two user groups, considering the mobile cloud computing features, user experience and user perception theory, build a mobile cloud computing users to evaluate the impact of individual and corporate users factor model evaluation system for mobile cloud computing; and for the evaluation model developed mobile cloud computing system and the individual user environment factors and evaluate the core business users evaluate short board; were constructed by mobile cloud computing and empirical individual users and business users to optimize the model, cloud providers find and assign users to optimize cost rationalization equilibrium point. But for a certain kind of business mobile cloud computing optimization model and optimization algorithm based on no research involved, so for mobile Internet features, consider transfer calculations of distance education operations and scheduling features, an optimization model is established based on the mobile Internet and cloud efficient calculation of distance education system, with academic and practical significance. At the same time, a good user experience and user perception of distance education system will give cloud providers, and distance education institutions have a positive impact, it will enhance the credibility of mobile cloud computing services, and enhance the soft power of distance education, enhance user stickiness and satisfaction and to improve the profitability of cloud providers; the bad perception of the user and the user experience will give cloud providers have a negative influence will undermine the credibility of mobile cloud computing services, reduce stickiness and user satisfaction. Mobile cloud computing providers, in order to attract users and enhance user stickiness, the user must take full account of user perception and experience, and focus on the core factors. And the need to consider moving providers and distance education institutions for distance education system operating costs, optimizing individual and business users two dimensions, the actual establishment of individual users mobile cloud computing model of evaluation factors and business user evaluation system, research users perception and experience and evaluated, is of great significance for the development of mobile cloud computing. It helps mobile cloud computing providers 
clearly recognize the advantages and disadvantages of the services provided for the further development of mobile cloud computing and improve the decision-making basis.

\section{Related Works}

\subsection{Mobile Internet Cloud Computing}

Since the Open Gardens proposed the concept of mobile cloud computing, the academic world be a lot of research. Some scholars consider cloud service delivery delay data to the cloud caused by the impact of mobile users, computing resources, deploy and manage applications and services were studied. [9] For the best personalized service and information management problem of mobile cloud computing environment, we propose a context management model, which is to effectively manage resources through contextual information and provide users with the appropriate services. Mao and other P2P heterogeneous cloud services and mobile network convergence scenario presents a cloud-oriented document management system that uses mobile applications to transparently access ways to effectively improve the performance of cloud services. Kallasi and other wireless channels, fiber and mobile cloud data is also composed of a computing system, consider vendors to provide cloud computing services in the characteristics of the globe, a new cloud-based Context response program. In order to reduce the delivery time of the application data to meet application performance requirements, Mahade proposed a cloud cell, the cell cloud formation resource pool in the area close to the user and provides the user VM, can seamlessly handle compute-intensive applications, reducing application delivery time data. Document [9] for mobile applications presents a resource-intensive application-aware management program, which can be in a dynamic network environment by optimizing the resources of the mobile terminal, the cloud and the cloud between the cell to maximize the customer experience. Liang and other cloud services for mobile cloud computing scene geographically distributed, based on semi-Markov decision process presents a clever decision service program, which migrate through the service way to balance the computational load in different areas, which can effectively reduce termination rate improve system efficiency. Santan and other mobile cloud computing resources dynamically provisioning scenarios, an adaptive resource provisioning model, which uses forecast resource needs ways to prepare in advance for the user computing resources, can maintain a good quality of service, while improving resource utilization. Research and management of computing resources and deploy applications and services, to further reduce the network load and mobile cloud computing service delivery delay data systems is an important future research directions. Some scholars consider steaming ring to limit mobile application performance caused by the radio resource to the mobile cloud radio resource allocation were studied. Andreas mobile cloud computing environment presents an intelligent access control scheme, and for the realization of the program has developed a mobile cloud access controller, the program according to the user's location, the service requested information to intelligently access management. Sudip Misra for the user to switch the scene, according to the auction mechanism proposed transfer and reallocation of bandwidth algorithm to guarantee the quality of service by the user to switch the user's bidding strategy. Niyato Dusit etc. for multiple vendors to provide network and computing resources of the scene, using the bandwidth and computing resources, joint approach based on cooperative game presents a resource supply strategy ${ }^{[10]}$.

US National Institute of Standards and Technology from the user experience point of view, the cloud computing service model into infrastructure as a service, platform as a service, software as a service. Cloud computing services focus of current research is how to measure how users adopt cloud computing costs, and service providers to maximize the use of its physical resources. Cloud computing infrastructure users hoping to meet 
demand for quality of service provided to minimize payment. For cloud computing infrastructure provider, the provider needs according to the requirements in a timely manner to each application optimized dynamically allocated on the basis of an appropriate amount of resources, we must also meet the conditions for optimal overall efficiency. Mobile cloud computing has the following advantages: 1. get rid of the phone hardware. Phone and machine processing capacity large difference when dealing with big data, mobile terminal hardware is the biggest constraint. But in the mobile cloud computing, data storage and computing capabilities to users from the "cloud", so it is no longer dependent on their mobile terminal processing computing power. 2 . quick and easy data access. Because mobile cloud computing data stored on the "cloud", the mobile cloud computing enables users with large data storage space, and when users access the cloud data in a mobile network environment can achieve good local access speed, and to facilitate inter-user data sharing. 3. Intelligent load balancing. Mobile cloud computing by utilizing change cycle between multiple applications, intelligent load balancing for the load changes in the larger applications to provide users with the flexibility to resources. 4. Reduce administrative costs. Management of resources and management costs proportional. Mobile cloud computing can be implemented standardized management processes, enabling management tasks clear, rationalizing management costs. 5. Press the user needs to provide services. Although customized services to reach a different Internet service users individual requirements, but will bring ultra-load bearing problems. The use of mobile cloud computing technology to share resources between different services, to provide users with on-demand service, so that reduce service costs. Mobile cloud computing there are some problems. Lack of resources the mobile terminal device. Relative to desktop computers, mobile terminals smaller screen, the battery capacity is limited, and the computing power of the device itself is not very strong, it is often considered mobile cloud computing cloud is that the user to thin clients and browsers as a medium for Cloud Access perform data processing and computing in the cloud. By mobile network Quality. Despite the reduction in the delay, but in the presence of multiple mobile devices at the same time reduces the bandwidth. At the same time, the bandwidth for mobile phones may be restricted in some area of base station bandwidth, there may be disconnected after operators optimize mobile network connection is interrupted and Suman situation will improve, but the mobile network environment the complexity of the connections may still be unstable and Suman phenomenon. Mobile terminal safety problems exist. Because more mobile devices than the desktop computer is lost, if the cloud platform can also access important data through the device to other users, it will bring information security risks. Heterogeneous wireless networks. Due to the rapid advances in wireless communication technology and network technology, resulting in a lot of new heterogeneous networks, such as cellular mobile communication network, wireless personal area networks, wireless LANs, wireless metropolitan area networks, wireless sensor networks.

\subsection{Internet Education System}

Mobile Learning research project was first started at Carnegie Mellon University. At present, mobile learning abroad mainly in the part of the developed countries in Europe and North America, and its applications have been fully carried out, related to the field of primary and secondary schools, university fields, vocational training, distance education, education for the whole of society . A total of three stages, the first stage, the feasibility of mobile learning and to verify the validity of the exploration stage. From 2001 to 2002 the company launched mobile learning project. Project goal is a systematic study of mobile terminal equipment applications in the classroom teaching, the research is to determine the main mobile terminal device for the secondary classroom teaching whether it is useful to explore the prospects for mobile learning undertaken. From the findings of the project, the teachers on the use of handheld devices aided teaching in the classroom is a very 
positive attitude, about $90 \%$ of the experimental teachers think that the handheld device is useful adjunct teaching tool can effectively promote students' classroom learning. The European Commission-funded project was launched in the United Kingdom since, on the feasibility study of mobile learning provides empirical data. The project is aimed at young unemployed or difficult years of education in the UK for the study, these people need education, but refused to enter school or fixed place of learning, the researchers found that this type of research by groups of young people have mobile phones, so consider to provide educational content based mobile learning mode. The study showed that after $62 \%$ of students through training mobile learning, has greatly improved the level of knowledge and enhance interest in learning, further research and application of the research results of mobile learning provides a good prerequisite. The second phase of mobile learning theory and application of model exploration stage - Mobile Learning and education resources integration. It can effectively support collaborative learning in a wireless learning environment, especially the collaborative knowledge construction. These projects are the use of mobile technology, the characteristics of the various types of mobile learning with learning mode integration, such as: problem-based learning, informal learning and collaborative learning. British African savannah learning project explores the use of mobile devices and wireless technology to achieve a rich, interactive experience simulated learning experiences in order to promote students' knowledge construction; Japan's University of Tokushima in Japan in Politeness expression pervasive learning system can automatically obtain learner position and push for polite language of the scene based on the learner's scene. Such research projects are carried out learning by providing mobile technology to support learning in real situations, more conducive to learners construct new knowledge-based mobile technology support for the scene. In recent years, in conjunction with mobile communications technology and social software for mobile learning has become a new hotspot. European project has developed a system for mobile terminals and the client, users can access other users to share learning content through this system, you can always publish text, pictures, learning experience to share with others; University of Nottingham has developed a platform for learners You can use the phone to learn experience sent to the personal space of the platform will be shared with other people in the museum, they can also see what others are gathering information to learn, the teacher finally student works selected outstanding posted to the website, to share to other learners, parents and the public; cloud computing concept was first proposed, a few years has been rapid development, now cloud computing has penetrated into all fields of study. In recent years, academic and commercial areas of cloud computing in education is very promising. Cloud computing aided teaching research project after another, including research in the cloud computing environment is one of the hottest mobile learning direction. Through a variety of educational services for the cloud smartphone, the learners can be more convenient for mobile learning. The third stage, the mobile learning app promotion stage. With the further expansion of mobile learning research and application of the scale, and gradually formed based on knowledge transfer and feedback; support mobile learning scenarios learning; individual learning mobile technical support; mobile technology-supported collaborative learning and social learning and other typical application mode. People began to move learning teaching model to promote large-scale study to construct the theoretical framework of regional mobile learning research carried out, for example, et al proposed a framework for mobile learning research conducted on campus to promote mobile learning in schools at all levels, community, national within the scope of conduct; to advance the field of education to have one share, the United States hundreds of basic education schools have joined the 1: 1computer project. MIT professor Nicholer sponsored alliance to explore and support research focusing on mobile learning terminal device form, make efforts to promote the goals of the world's children to have one. International Distance Education Authority said: "The technology itself is not suitable for teaching characteristics, making distance education and open university has 
been successful, but the technology has been commonplace in public." Moving from the current situation of foreign research study, the good proof of this statement. Mobile learning is experienced efficacy studies, terminal support research and new applications in the form of an integrated, large-scale promotion of applied research in three phases and has a generally recognized by the public only after the path of rapid development.

Mobile learning as an emerging technology in the field of education research, current research has not yet formed its concepts more unified concept of consensus, many mobile learning Researchers have there are different views and opinions, it is because the elements involved in mobile learning more, such as: Do you want to focus on the definition of small personal devices, whether mobile learning to pay attention to learners, whether you want to focus on communication and collaboration between learners, whether to pay attention to the context associated with the learner, if you want to focus on expansion of online learning. Mobile learning is a mobile communications technology and computer network learning and development combined with the product. On the one hand, intelligent, portable and mobile devices is seen as an extension of digital learning technologies, on the other hand, multimedia mobile learning, mobile devices, haptic interaction, intelligent environments, wireless communications and other new technologies into the field of education and training Therefore, mobile learning exhibits and general computer-based learning traditional fixed wired network model under different digital learning characteristics. Small volume portable learning devices. With the rapid development of mobile terminal equipment, miniaturized, intelligent mobile terminal has become quite common functions are quite powerful, which provides learners with a variety of learning tools, such equipment outstanding feature is convenient, flexible, and different learning modes larger host. Flexible and independent learning time, learning spaces without space restrictions. Learners carry small mobile terminal devices, you can now access learning system through a wireless network to carry out anytime, anywhere learning, which allows learners to completely get rid of the shackles of geographical location at any time at their own comfortable and flexible learning environment. In addition, due to the different goals of each learner and learning tasks, so that they can learn to move according to their own learning needs and learning willingness to customize the arrangement of learning content and learning time. More situational learning activities, resources, rich, and real-life situation as a learning metaphor. Mobile learning provides contextual learning support, learning activities take place in the true nature of the social situation, the learner to facilitate learning and practice environment, which makes it more abstract learning content specific, so that students understand; in addition, there are also learning scenarios help learners to apply what they have learned to live among actual production, namely to achieve a "live learning" in the true sense. Broader learning community and lifelong learning process. Emphasis on learning ability in today's society, leaving the school does not mean the termination of learning activities. Therefore outside the classroom informal learning activities will always exist. More learners do not have time because of work or other reasons to attend the formal return to the classroom learning and give up the opportunity of continuing education. Emergence of mobile learning allows a wider range of people to participate in learning. The majority of learners can easily go to the browser they want to learn content through a wireless network, quickly download the information they want. So mobile learning broadens the scope of education, so that more people access to education, to achieve the current advocacy of lifelong education.

\section{The Proposed Scheme}

Based distance education system for mobile cloud by six layers and two service modules, of which 6 layers are data sources, data integration, data storage, data analysis and processing, information presentation and business applications, two service modules 
to provide security for the entire system services and access control services.

1) Data source

The system supports multiple types of data source access, mainly include two categories: The first category is related to distance education and business data, including user-demand services, search data, text data, education, education and other audio and video data; the second category external data, including authentication, recommendations, comments and other data, the system to obtain such data from authoritative data distribution mechanism.

\section{2) Data Integration}

This layer is formed by parallel ETL technology data source layer by extraction, transformation, loading and a series of processes is loaded into the data storage layer. For different types of data sources we offer different ETL interface, and the degree of parallelism ETL process can be set as desired.

\section{3) Data Storage}

Data storage system in the cloud data warehouse, data warehouse uses a distributed cloud storage technology to meet the massive data storage requirements, not only safe, reliable, and economical, easy to expand. In order to meet the user real-time and non-real-time data processing needs, cloud data warehouse is divided into two parts: ODS and distributed data warehouse, where the ODS to meet the storage needs of data in real-time, distributed data warehouse to meet the non-real-time data storage requirements. To meet the specific needs of data exchange between the ODS and the distributed data warehouse via the interface.

4) Data analysis and processing

Data analysis and processing of data in the cloud layer data warehouse provides three types of processing: ad hoc queries, multi-dimensional OLAP analysis, data mining and machine learning. Ad hoc queries to meet the needs of user queries for real-time data, ODS part of the data stored in the data storage layer; multi-dimensional OLAP analysis, multi-dimensional analysis based on parallel computing technology to meet user demand for OLAP analysis of massive data; data mining and machine learning to provide clustering, forecasting, outlier analysis, correlation analysis and other types of parallel data mining algorithms, allowing users to tap unknown, valuable knowledge from vast amounts of data, thus providing a basis for decision making by managers.

5) information be presented

Information presentation layer mainly simple appearance, landscaping FusionCharts charting tools as the underlying display mode, the dynamic display indicators and parameters, including the form of a histogram showing a variety of charts, pie charts, line graphs, real-time maps, scale diagrams.

\section{6) business applications}

Business application layer provides multiple levels of education for intelligent remote user applications, including online cloud notes, mastery of analysis, ability to use analysis and user behavior analysis and so on.

\section{7) Service Module}

System service module provides security and access control services for the system. Security should be application security, authentication mechanism, application security auditing, data storage confidentiality, data storage integrity, non-repudiation, software fault tolerance, resource control, backup and peer recovery application data aspects of 
security; access control services for system resources providing flexible rights allocation mechanism, while ensuring the safe use of system resources.

\subsection{Cloud Data Processing Platform}

Cloud data processing platform is the core of the system of data storage and data processing platform, the platform structure to a distributed file system and parallel computing framework, integration interfaces to external systems, multi-dimensional analysis of massive data knowledge mining, data analysis and other results show service, as shown below, the system can support large-scale distributed data acquisition, multi-dimensional analysis of parallel and parallel data mining.

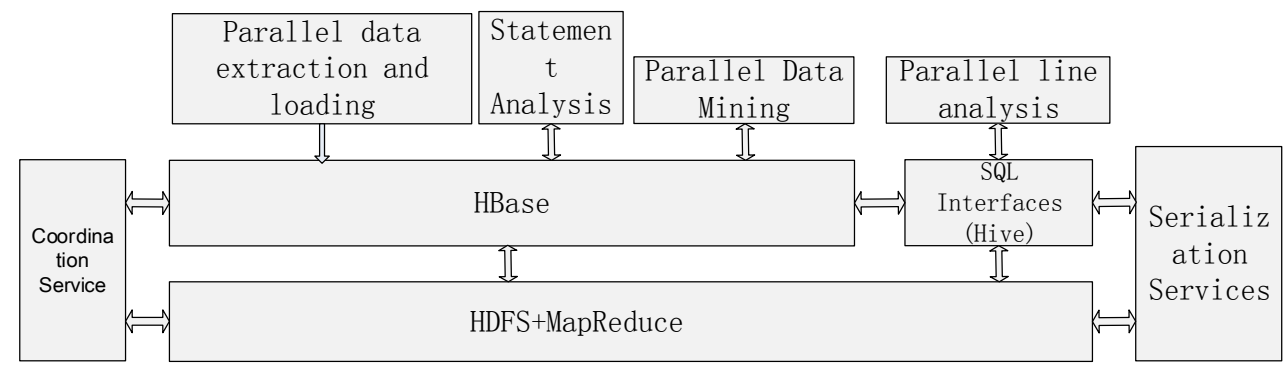

Figure 1. Data Relationships

As can be seen from Figure 1, the main provider of cloud data processing platform features four categories, which are distributed data storage, parallel data extraction, transformation and loading (ie, data ETL), parallel and parallel data mining multi-dimensional analysis. Wherein the distributed data storage uses HBase to store data, which can greatly improve the efficiency of data access, since the underlying storage HBase uses HDFS, so that a distributed data storage, safe and reliable. ETL data used to complete the data source through the extraction, transformation, loading process into a series of HBase. Parallel to achieve multi-dimensional analysis using Hive, class Hive SQL interface provides easy to use, multi-dimensional analysis greatly simplifies the difficulty of implementation. MapReduce parallel data mining based on parallel computing framework to achieve clustering, classification, prediction, outlier analysis and other data mining algorithms make it possible to discover valuable knowledge from massive data.

Cloud data processing platform in parallel multidimensional analysis technology uses Hive achieve the specific implementation steps is the first to map data stored in HBase or HDFS to the Hive, and then type SQL interfaces Hive provides data multidimensional analysis, Hive class SQL statements into MapReduce Job executed in parallel, enabling the entire multi-dimensional analysis of parallelization.

In this system, the parallel multi-dimensional analysis technique is mainly used in various electrical analysis and comprehensive analysis of energy efficiency, energy use by a variety of indicators of users multi-dimensional analysis enables users of electricity and energy consumption at the level of the multi-angle statistics and evaluation, to provide users with decision support. According to the specific needs of intellectual distance education system, the initial user data parallel multi-dimensional analysis of the main users to learn query data, analysis dimension has user type, time, location, related topics, learning routes.

In this system, data mining is mainly used for electricity a lot of basic data analysis and processing and converting it into valuable knowledge and conclusions, these conclusions reflect the knowledge and the laws of electricity and energy users weaknesses can be auxiliary power grid companies and government departments decision-making, but also the power user guide rational use of electricity.

For general data mining massive, high-frequency data study, the prevalence of single 
algorithm to achieve a huge amount of calculation, calculation process is complex, limited processing power and other issues. In order to solve the problems of conventional data mining algorithms encountered in stand-alone operation, improve operating efficiency, the use of parallel computing framework MapReduce cloud computing to achieve clustering, classification, prediction, association and other data mining algorithms parallelization and apply in distance education learners to acquire knowledge and learning behavior associated analysis. Cloud data processing platform parallel the functional structure of the data mining module.

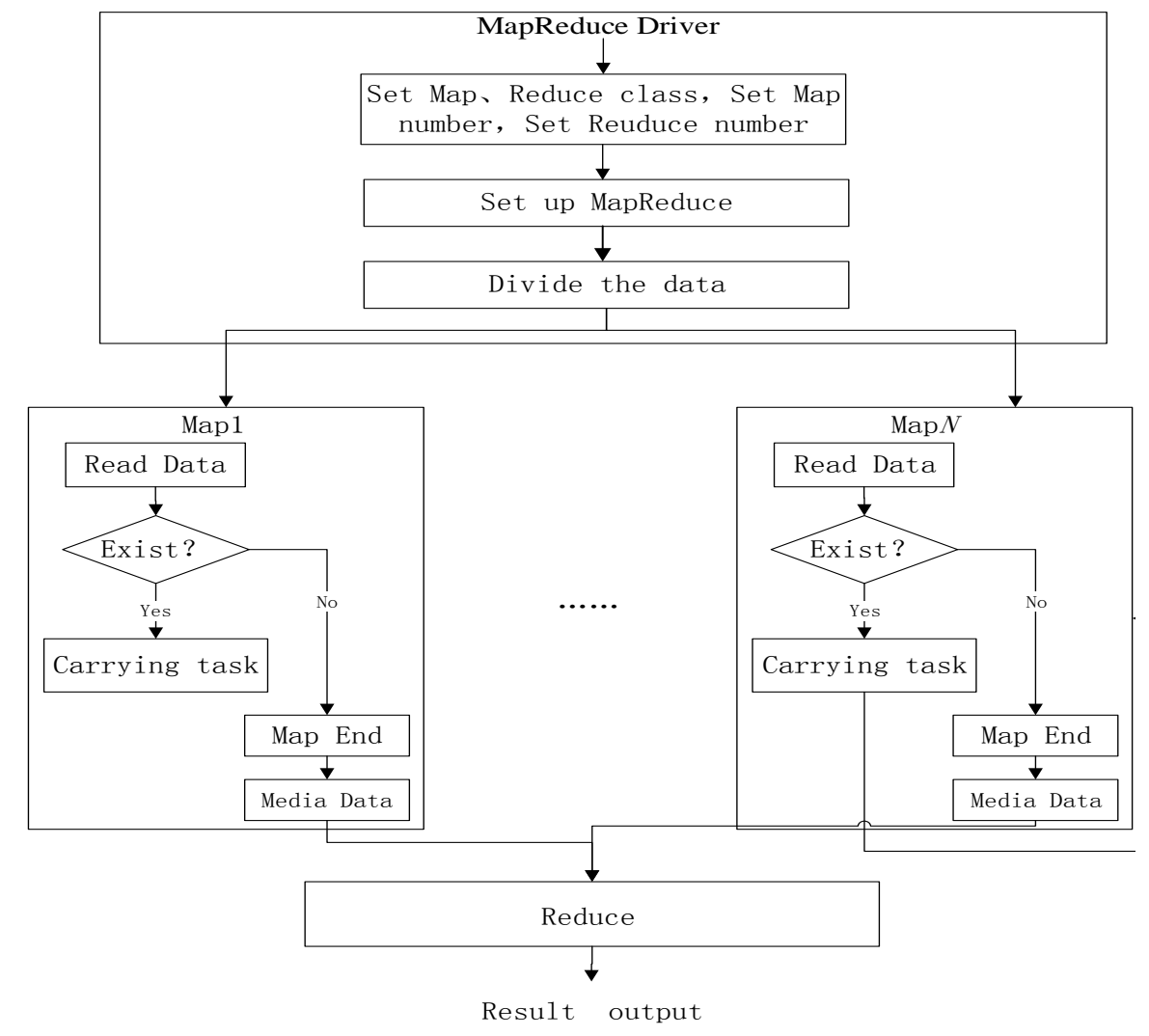

Figure 2. MapReduce-based Data Mining Parallel Algorithm Flow Chart

\section{Smart Interactive}

Through intelligent interactive system, the educational institutions, distance education providers, interaction and sharing of information between the remote user education, relevant party can enjoy information inquiry, under the information is sent, information consulting and other flexible interactive services. Smart interactive features include remote user education, information exchange service, system interaction services.

1) Distance Education Information Query

This feature enables remote users to education, educational resources and learning search path information management functions, providing users to view education learning history related information and related course materials inquiries. Conditions can be viewed by searching the whole information of distance education information about related topics, inquiry learning materials and information mutual relations, as well as relevant learning users reviews, etc.

2) information exchange service

Information exchange services to provide remote education users query search of educational resources and learning path published information, modify unpublished information, delete unpublished information, publishing new information and other 
operations, such as updating the curriculum, the new start of the course and other information, distance education agency released study suggested user messages and other information. In addition, information exchange service to support fuzzy queries, provide energy efficiency information query, add, modify and delete services.

3) systems for interactive services

According to business needs, and other applications for data exchange and data sharing. System main function is to use interactive services data bus, the data bus services to other applications within the system and outside the system in acquisition, the resulting data is applied to this system. In this system, mainly through the interactive system integration services to solve problems with other business systems. Based interface-oriented thinking in the design and third-party service interface, unified by the interface processing, using an adapter design pattern of thought, to complete the conversion process by the middle of no gap between systems integration.

Security applications should apply system security, authentication mechanism, user rights and access control, application security auditing, data storage confidentiality, data storage integrity, software fault tolerance, resource control, backup and recovery and other aspects of the application data security.

The user interface for Safety Security users to connect remotely applications, including authentication, need to develop data encryption, access control and other security measures, and the use of encryption technology to ensure the integrity of the data communication process.

Data interface between systems security including security of data exchanged between the Intelligent Community Business Intelligence system interfaces with other business systems, such as data exchange interfaces with third-party marketing business system information collection system. Sharing and exchanging data between systems used in two modes:

(1) interface to exchange data directly between systems.

(2) by security isolation gateway for data exchange.

\subsection{K-Means Algorithm}

KMeans algorithm is a very simple clustering algorithm based on the distance that each Cluster and this similarity is measured by the distance from the point of similar composition, the point between the different Cluster should try not similar, and each will have a Cluster "center of gravity"; in addition, it is also an exclusive algorithm that at any point must belong to a Cluster and only belong to the Cluster. Of course, its shortcomings are more obvious, such as: For the isolated point sensitive to the size of the gap between the final cluster produce little.

It is an iterative algorithm:

1) according to pre-given value of $\mathrm{k}$ establish initial division, get $\mathrm{k}$ Cluster, for example, a random selection of $\mathrm{k}$ points as the $\mathrm{k}$ Cluster's center of gravity, or with Canopy Clustering obtained Cluster as the initial focus (of course, this time of $\mathrm{k}$ Clustering results obtained value is determined by the Canopy);

2) calculated for each point to the center of gravity away from each Cluster, add it to the nearest Cluster;

3) Re-calculated for each Cluster's center of gravity;

4) Repeat procedure 2-3 until each Cluster does not change the center of gravity or the maximum number of iterations to achieve within a certain range of accuracy.

The time complexity of the algorithm is: $\mathrm{O}(\mathrm{nkt})$, where: $\mathrm{n}$ is the number of cluster points, $\mathrm{k}$ is the number of Cluster, $\mathrm{t}$ is the number of iterations.

Parallel strategy:

K-Means better Locality it can well be parallelized. The first stage, the process of generating Cluster can be parallelized, the presence of each Slave read local data sets generated by the above algorithm Cluster collection, and finally set a number of Cluster 
generate a first iteration of the Global Cluster collection, and then repeat the process until satisfied end condition, the second stage, with a cluster operation before getting the cluster.

With map-reduce description: datanode in map stage read in the local data set, the output of each point and its corresponding Cluster; combiner operating in a local included in the same Cluster in points reduce operation and output, reduce operations to give global Cluster collection and write HDFS.

Take advantage of parallel clustering algorithm, cluster analysis, distance education institutions in order to classify the user to achieve classification of service, more targeted, and ultimately individual learning tailored.

\section{Conclusion}

Based on the characteristics of distance education, presented in the new technological environment of mobile Internet cloud conditions, building distance education system for mobile cloud computing, according to the computing power of the mobile terminal is poor, but the access convenient features, build distance education query, data output, and clustering data mining technology platform for mobile cloud platform, thereby enabling the characteristics of distance education system supports multiple services.

\section{References}

[1] H. Ye, "Optimization of Resource Scheduling Based On Genetic Algorithm in Cloud Computing Environment", (2015).

[2] W. Li, Y. Zhao and S. Lu, "Mechanisms and challenges on mobility-augmented service provisioning for mobile cloud computing", Communications Magazine, IEEE, vol. 53, no. 3, (2015), pp. 89-97.

[3] I. A. T. Hashem, I. Yaqoob and N. B. Anuar, "The rise of "big data" on cloud computing: Review and open research issues", Information Systems, vol. 47, (2015), pp. 98-115.

[4] M. Sookhak, A. Gani and H. Talebian, "Remote data auditing in cloud computing environments: a survey, taxonomy, and open issues", ACM Computing Surveys (CSUR), vol. 47, no. 4, (2015), p. 65.

[5] T. Mastelic, A. Oleksiak and H. Claussen, "Cloud computing: survey on energy efficiency", ACM Computing Surveys (CSUR), vol. 47, no. 2, (2015), p. 33.

[6] V. R. Pancholi and B. P. Patel, "Enhancement of Cloud Computing Security with Secure Data Storage using AES", International Journal for Innovative Research in Science and Technology, vol. 2, no. 9, (2016), pp. 18-21.

[7] R. A. Phipps, "Measuring Quality in Internet-Based Higher Education", International Higher Education, vol. 20, (2015).

[8] R. Wegerif, "Toward Dialogic Literacy Education for the Internet Age”, Literacy Research: Theory, Method, and Practice, vol. 64, no. 1, (2015), pp. 56-72.

[9] J. Liddle and P. Liang, "Mixed evidence exists for Internet-based education and support interventions for caregivers of someone with a chronic health condition in enhancing wellbeing and decreasing stress", Australian Occupational Therapy Journal, vol. 63, no. 2, (2016), pp. 137-138.

[10] K. Harikumar, S. Gopinath and A. Raghunath, "Computers and internet in dental education system of Kerala, South India: A multicentre study", Journal of the International Clinical Dental Research Organization, vol. 7, no. 1, (2015), p. 82.

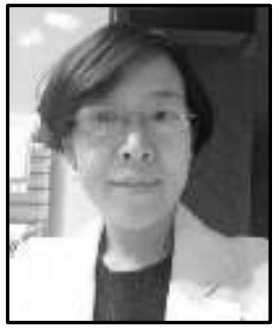

\section{Author}

Junying Liu, Applied Mathematics Master,Xiang Tan University Work on the research and operation management of enterprise staff education and training, the design of large scale enterprise network university planning distance education system. 
International Journal of Grid and Distributed Computing

Vol. 10, No. 1 (2017) 